\title{
NMR STUDIES OF TRANSLATIONAL MOTION
}

Translational motion in solution, either diffusion or fluid flow, is at the heart of chemical and biochemical reactivity. Nuclear magnetic resonance (NMR) provides a powerful non-invasive technique for studying the phenomena using magnetic field gradient methods. Describing the physical basis of measurement techniques, with particular emphasis on diffusion, balancing theory with experimental observations and assuming little mathematical knowledge, this is a strong, yet accessible, introduction to the field. A detailed discussion of magnetic field gradient methods applied to magnetic resonance imaging (MRI) is included, alongside extensive referencing throughout, providing a timely, definitive book to the subject, ideal for researchers in the fields of physics, chemistry and biology.

William S. Price is Professor and Chair of Nanotechnology in the School of Biomedical and Health Sciences and the School of Medicine, University of Western Sydney. He directs the Biomedical Magnetic Resonance Facility and leads the Nanoscale Organisation and Dynamics Group, University of Western Sydney. Professor Price is an expert in the theory, development and application of NMR spectroscopy, focussing on NMR diffusion measurements and MRI techniques for studying molecular association and molecular dynamics. 


\title{
Cambridge Molecular Science
}

As we enter the twenty-first century, chemistry has positioned itself as the central science. Its subject matter, atoms and the bonds between them, is now central to many of the life sciences on the one hand, as biological chemistry brings the subject to the atomic level, and to condensed matter and molecular physics on the other. Developments in quantum chemistry and in statistical mechanics have also created a fruitful overlap with mathematics and theoretical physics. Consequently, boundaries between chemistry and other traditional sciences are fading and the term Molecular Science now describes this vibrant area of research.

Molecular science has made giant strides in recent years. Bolstered both by instrumental and by theoretical developments, it covers the temporal scale down to femtoseconds, a time scale sufficient to define atomic dynamics with precision, and the spatial scale down to a small fraction of an Angstrom. This has led to a very sophisticated level of understanding of the properties of small molecule systems, but there has also been a remarkable series of developments in more complex systems. These include protein engineering; surfaces and interfaces; polymers; colloids; and biophysical chemistry. This series provides a vehicle for the publication of advanced textbooks and monographs introducing and reviewing these exciting developments.

\author{
Series editors \\ Professor Richard Saykally \\ University of California, Berkeley \\ Professor Ahmed Zewail \\ California Institute of Technology \\ Professor David King \\ University of Cambridge
}


Cambridge University Press

978-0-521-80696-1 - NMR Studies of Translational Motion

William S. Price

Frontmatter

More information

\title{
NMR STUDIES OF TRANSLATIONAL MOTION
}

\author{
WILLIAM S. PRICE \\ Nanoscale Organisation and Dynamics Group
}

University of Western Sydney, Australia 
CAMBRIDGE UNIVERSITY PRESS

Cambridge, New York, Melbourne, Madrid, Cape Town, Singapore, São Paulo, Delhi

Cambridge University Press

The Edinburgh Building, Cambridge CB2 8RU, UK

Published in the United States of America by Cambridge University Press, New York

www.cambridge.org

Information on this title: www.cambridge.org/9780521806961

\section{C) W. S. Price 2009}

This publication is in copyright. Subject to statutory exception and to the provisions of relevant collective licensing agreements no reproduction of any part may take place without

the written permission of Cambridge University Press.

\section{First published 2009}

Printed in the United Kingdom at the University Press, Cambridge

A catalogue record for this publication is available from the British Library

Library of Congress Cataloguing in Publication data

Price, William S., 1964-

NMR studies of translational motion / William S. Price.

p. $\mathrm{cm}$.

1. Translational motion - Measurement. 2. Reactivity (Chemistry).

3. Nuclear magnetic resonance. 4. Diffusion and 5. Magnetic resonance imaging. I. Title.

$$
\begin{gathered}
\text { QC762.6.T73P75 } 2008 \\
\text { 541'.39-dc22 } \\
\text { 2008027008 }
\end{gathered}
$$

ISBN 978-0-521-80696-1 hardback

Cambridge University Press has no responsibility for the persistence or accuracy of URLs for external or third-party internet websites referred to in this publication, and does not guarantee that any content on such websites is, or will remain, accurate or appropriate. 


\section{Contents}

Preface ix

Acknowledgements xiv

Abbreviations $\quad \mathrm{xv}$

1 Diffusion and its measurement 1

1.1 Introduction 1

1.2 Types of translational motion - physical interpretation and significance

1.3 Mathematical modelling of self-diffusion 6

$\begin{array}{ll}1.4 \text { Propagation } & 14\end{array}$

$\begin{array}{lll}1.5 & \text { Solving the diffusion equation } & 20\end{array}$

1.6 Mean square displacement, time-dependent diffusion coefficients and the diffusion ellipsoid $\quad 31$

$\begin{array}{lll}1.7 & \text { Measurement timescales and restricted diffusion } & 36\end{array}$

1.8 Diffusion in heterogeneous, porous and polymer systems $\quad 39$

1.9 Flow in porous media $\quad 54$

1.10 Techniques for measuring diffusion $\quad 55$

$\begin{array}{ll}\text { References } & 57\end{array}$

2 Theory of NMR diffusion and flow measurements $\quad 69$

2.1 Introduction $\quad 69$

2.2 Nuclear spins, magnetic gradients and motion $\quad 70$

2.3 Correlating the PGSE signal attenuation with diffusion 90

2.4 Oscillating gradients, higher-order gradient pulse trains and probing $D_{\omega}(\omega) \quad 98$

2.5 PGSE of freely diffusing multicomponent systems 101

2.6 The long-range dipolar field and multiple echoes 111

$\begin{array}{ll}\text { References } & 112\end{array}$

3 PGSE measurements in simple porous systems $\quad 120$

$\begin{array}{ll}3.1 \text { Introduction } & 120 \\ 3.2 & 120\end{array}$

3.2 Experimental determination of $\bar{P}(\mathbf{R}, \Delta) \quad 122$ 
Cambridge University Press

978-0-521-80696-1 - NMR Studies of Translational Motion

William S. Price

Frontmatter

$\underline{\text { More information }}$

3.3 Experimental determination of the moments of $\bar{P}(\mathbf{R}, \Delta) \quad 122$

3.4 The spectral function and the return to origin probability 124

$\begin{array}{ll}3.5 & \text { Diffusive diffraction } \\ 3.6 & 125\end{array}$

3.6 An example - diffusion between planes 127

$\begin{array}{lll}3.7 & \text { Diffusion in reflecting spheres } & 130\end{array}$

3.8 Size distributions of the restricting geometry 131

3.9 Finite gradient pulses and the validity of the SGP and GPD $\begin{array}{ll}\text { approximations } & 132\end{array}$

3.10 Other methods for determining $E(\mathbf{q}, \Delta) \quad 135$ References 141

4 PGSE measurements in complex and exchanging systems 147

$\begin{array}{lll}4.1 & \text { Introduction } & 147\end{array}$

4.2 Simple binding and exchange: the Kärger equations 150

4.3 Exchange between free and restricted sites 155

4.4 Anisotropic and low dimensional diffusion 163

$\begin{array}{ll}4.5 \text { General porous media } & 170\end{array}$

$\begin{array}{lll}4.6 & \text { Polymer diffusion } & 173\end{array}$

$\begin{array}{lll}4.7 & \text { Flow and velocity distributions } & 174\end{array}$

$\begin{array}{lll}4.8 & \text { Internal magnetic field gradients } & 175\end{array}$

$\begin{array}{ll}\text { References } & 177\end{array}$

5 PGSE hardware 185

$\begin{array}{lll}5.1 \text { Introduction } & 185\end{array}$

5.2 Gradient coils and fringe fields 186

5.3 Current amplifiers 191

5.4 Connecting to the spectrometer 192

5.5 Field-frequency locking 193

5.6 Temperature control and calibration 193

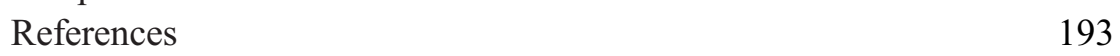

6 Setup and analysis of PGSE experiments 198

$\begin{array}{ll}6.1 \text { Introduction } & 198\end{array}$

6.2 Selection of PGSE parameters 199

6.3 Sample preparation 204

$\begin{array}{ll}6.4 \text { Gradient calibration } & 205\end{array}$

6.5 Analysis 212

References $\quad 215$

7 PGSE hardware and sample problems 221

$\begin{array}{lll}7.1 \text { Introduction } & 221\end{array}$

$\begin{array}{lll}7.2 & \text { RF problems } & 221\end{array}$

7.3 Radiation damping and long-range dipolar field effects 222

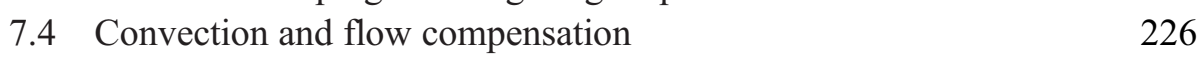

$\begin{array}{lll}7.5 \text { Gradient constancy } & 229\end{array}$ 
7.6 Background gradients

7.7 Eddy currents and perturbation of $B_{0}$

7.8 Gradient mismatch and sample movement

7.9 Cross relaxation

7.10 Homonuclear scalar couplings

8 Specialised PGSE and related techniques 256

$\begin{array}{lll}8.1 \text { Introduction } & 256\end{array}$

8.2 Steady gradient and stray field measurements 256

8.3 Multiple quantum and heteronuclear 258

$\begin{array}{lll}8.4 & \text { Fast sequences } & 262\end{array}$

8.5 Distributions of relaxation times 269

8.6 Multi-dimensional mixture separation and diffusion editing 269

8.7 Double PGSE and multi-dimensional correlations 272

$\begin{array}{ll}8.8 \text { Flow measurements } & 281\end{array}$

8.9 Electrophoretic NMR 282

8.10 Multiple spin-echoes and miscellaneous $\quad 286$

References 286

9 NMR imaging studies of translational motion 296

9.1 Introduction 296

9.2 Imaging - basic concepts 297

9.3 Combining PGSE measurements with imaging 297

9.4 Imaging-based flow measurements 300

References $\quad 303$

$10 \quad B_{1}$ gradient methods $\quad 308$

10.1 Introduction $\quad 308$

$\begin{array}{lll}10.2 B_{1} \text { gradients } & 308\end{array}$

$10.3 B_{1}$ gradient diffusion measurements $\quad 309$

$\begin{array}{ll}10.4 \text { Miscellaneous } & 310\end{array}$

References $\quad 311$

11 Applications $\quad 313$

$\begin{array}{ll}11.1 \text { Introduction and reviews } & 313\end{array}$

11.2 Applications to high-resolution NMR 314

11.3 Biological and pharmaceutical studies 316

$\begin{array}{ll}11.4 \text { Chemical and material studies } & 326\end{array}$

$\begin{array}{ll}11.5 \text { Spin-diffusion } & 340\end{array}$

11.6 Imaging-based studies $\quad 340$

References $\quad 344$

Appendix $\quad 369$

$\begin{array}{ll}\text { Index } & 377\end{array}$ 


\section{Preface}

Translational motion in solution (e.g., diffusion, flow or advection) plays a central role in science. Self-diffusion can be rightfully considered as being the most fundamental form of transport at the molecular level and, consequently, it lies at the heart of many chemical reactions and can even govern the kinetics. Diffusion, due to its very ubiquity, is encountered in a myriad of scientific studies ranging from diseases to separation science and nanotechnology. Further, the translational motion of a species not only reflects intrinsic properties of the species itself (e.g., hydrodynamics), but can also shed light on the surrounding environment (e.g., intermolecular dynamics or motional restriction). Consequently, being able to study and ultimately understand the translational motion of molecules and molecular systems in their native environment is of inestimable scientific value.

Measuring translational motion at the molecular level presents special difficulties since labelling (e.g., radiotracers) or the introduction of thermodynamic gradients (which leads to mutual diffusion and consequently irreversible thermodynamics) in the measurement process can have deleterious effects on the outcome. Also, in many instances it is of interest to measure the diffusion of species at quite high concentrations. Fortunately, nuclear magnetic resonance (NMR) provides a means of unparalleled utility and convenience for performing non-invasive measurements of translational motion. Of particular significance is that, in general, the species of interest inherently contain NMR-sensitive nuclei and thus sample preparation generally requires nothing more than placing the sample into the NMR spectrometer.

NMR is an inherently quantum mechanical subject, yet translational motion of molecules lies between the microscopic and the macroscopic and is most conveniently described with classical physics. Abiding by Ockham's razor, this is the path that will be taken here. This is not an NMR textbook per se, and for the fundamentals of NMR numerous excellent texts are currently available. ${ }^{1-6}$ A tabulation of many of the commonly used NMR acronyms can be found elsewhere. ${ }^{7}$ 
The last two decades have seen the advent of widespread commercial availability of MRI imagers and magnetic gradient probes and gradient drivers for NMR spectrometers. Magnetic gradients now pervade almost all areas of NMR ranging from coherence selection, gradient shimming, selective excitation and signal suppression $^{8-21}$ in high-resolution NMR to inputting spatial dependencies into NMR imaging (also known as MRI, and NMR microscopy) and NMR diffusion measurements. The use of gradient NMR allows diffusion to be added to the list of standard NMR observables.

This book grew out of two widely cited pedagogical papers that I wrote sometime ago on measuring diffusion with $\mathrm{NMR}^{22,23}$ using magnetic field (i.e., $B_{0}$ ) gradient methods. The overall aim of the book is rather modest: to present a clear overview of this field with particular emphasis on trying to impart a clear physical picture ideally such that the literature may become more accessible to readers interested in expanding their research into this field. Specifically, this book endeavours first to explain the physical basis of magnetic gradient-based NMR measurements of translational motion - especially diffusion measurements involving pulsed magnetic field gradients. Flow- and imaging-based measurements are closely related technically and theoretically and so some coverage of these kindred fields is presented. Secondly, to provide a clear correlation between experimental methodology and theoretical data analysis and the sorts of applications that are feasible. I have cited rather more references than is typical in a monograph to give the reader more chance of understanding the present text and also the opportunity to read further. I also liberally cite some textbooks that I found particularly lucid - especially in mathematical areas since many of the readers of this book may not come from a strong mathematics/physics background and to this end I also include the derivations of some of the more important equations. Although in general I have tried to maintain historical lineage, in some cases I have cited a more accessible reference for a concept than the original reference. As is so often the case, what started as my attempt to clearly explain an area of science became an exercise in trying to make myself really understand the area. This area of NMR application, theory and methodology has grown exponentially in the past three decades. Thus, it is neither possible nor reasonable to attempt anything approaching a comprehensive coverage; further the ordering of the material was made particularly difficult by many of the studies being justifiably included under more than one of the subheadings.

The outline of this book is as follows: Chapter 1 serves as an introduction to translational motion with special attention to diffusion and begins with some introductory remarks on the relationship between diffusion and chemical and biological phenomena, followed by a detailed exposition on its physical basis and how it can be mathematically modelled - these concepts will be heavily drawn upon in later chapters. This basis also allows the final section, where some of the more 
common techniques for measuring diffusion are briefly reviewed, to be placed in perspective as it allows the strengths and limitations of the different methods of diffusion measurements to be more clearly understood. Chapter 1 is rather solid and parts can be skipped on a first read or by those only interested in the NMR techniques and not the fundamental aspects of diffusion and its modelling.

Chapter 2 introduces the basic concepts behind magnetic gradient-based NMR diffusion measurements in freely diffusing systems and in particular the workings of basic pulsed gradient spin-echoes (PGSE), the most common (analytical and approximate) mathematical procedures for correlating the experimental variables, diffusion (and flow) with the observed NMR signal. Measurements of samples containing mixtures of species and aggregating species, but where the exchange is slow on the measurement timescale, are also considered.

Chapter 3 builds upon Chapter 2 and extends the coverage to the cases of simple porous systems, that is where a species is confined to a single highly symmetrical pore of some description. Whereas analytical relations could be given for the relationship between experimental variables and observed signal in the case of free diffusion, such analysis even for simple highly symmetrical pores rapidly becomes mathematically intractable and thus some consideration is given to the validity of the commonly used mathematical approximations and the various numerical approaches that are sometimes used.

Chapter 4 continues on from Chapter 3 and considers phenomena such as exchange, anisotropic and flowing systems and diffusion in polymers. Diffusion measurements in internal magnetic fields are also given brief mention.

Chapter 5 provides an overview of the additional instrumentation for an NMR spectrometer needed to conduct diffusion, flow and, by extension to a system capable of generating magnetic field gradients in three orthogonal directions, imaging-based measurements.

Chapter 6 details fundamental experimental considerations such as the selection of delays, gradient parameters and solvent suppression technique in diffusion measurements and how the applied gradient strength might be calibrated. This chapter ends with a section on how to transform and analyse the PGSE data resulting from simple freely diffusing samples, complex mixtures and samples involving restricted diffusion.

Chapter 7 concerns most of the issues that thwart attempts to get high-quality data such as background gradients, eddy currents induced by the rapidly pulsed magnetic field gradients and imperfectly generated gradient pulses. The symptoms and suggestions for obviating or at least meliorating their effects are considered.

Chapter 8 provides an overview of some of the more sophisticated sequences for measuring diffusion, flow and related phenomena.

Chapter 9 gives an overview of the inclusion of conventional nuclear magnetic resonance imaging (aka MRI) methodology with diffusion and flow measurements. 
Whereas in the previous chapters the systems being studied might be microscopically heterogeneous, there was an implicit assumption that they were macroscopically homogeneous. However, in many cases (biological tissues such as brain being a prominent example) the sample is also heterogeneous on a length scale that can be accessed by MRI methodology. The combination of MRI techniques with gradient-based diffusion and flow-measuring techniques provides powerful tools for characterising natural and synthetic materials.

Chapter 10 gives a brief overview of the use of radio frequency (i.e., $B_{1}$ ) field gradients to measure translational motion.

Chapter 11 surveys some of the applications to which gradient-based measurements of translational motion have been put.

\section{References}

1. R. R. Ernst, G. Bodenhausen, and A. Wokaun, Principles of Magnetic Resonance in One and Two Dimensions. (London: Clarendon Press, 1987).

2. J. W. Hennel and J. Klinowski, Fundamentals of Nuclear Magnetic Resonance. (Essex: Longman Scientific \& Technical, 1993).

3. D. Canet, Nuclear Magnetic Resonance Concepts and Methods. (New York: Wiley, 1996).

4. D. M. Grant and R. K. Harris (eds), Encyclopedia of Nuclear Magnetic Resonance. (New York: Wiley, 1996).

5. M. Levitt, Spin Dynamics - Basic Principles of NMR Spectroscopy, 2nd edn. (New York: Wiley, 2008).

6. J. Keeler, Understanding NMR Spectroscopy. (New York: Wiley, 2005).

7. D. D. Traficante, Abbreviations and Acronyms Used in Magnetic Resonance. Concepts Magn. Reson. 10 (1998), 59-62.

8. P. C. M. Van Zijl and C. T. W. Moonen, Complete Water Suppression for Solutions of Large Molecules Based on Diffusional Differences Between Solute and Solvent (DRYCLEAN). J. Magn. Reson. 87 (1990), 18-25.

9. D. M. Doddrell, Application of Pulsed Field Gradients in High-Resolution NMR Spectroscopy. J. Chin. Chem. Soc. (Taipei) 38 (1991), 107-17.

10. P. C. M. Van Zijl and C. T. W. Moonen, Solvent Suppression Strategies for In Vivo Magnetic Resonance Spectroscopy. NMR Basic Princ. Prog. 26 (1992), 67-108.

11. J. Keeler, R. T. Clowes, A. L. Davis, and E. D. Laue, Pulsed-Field Gradients: Theory and Practice. Methods Enzymol. 239 (1994), 145-207.

12. D. Canet and M. Décorps, Applications of Field Gradients in NMR. In Dynamics of Solutions and Fluid Mixtures, ed. J.-J. Delpuech. (New York: Wiley, 1995), pp. 309-43.

13. L. Mitschang, H. Ponstingl, D. Grindrod, and H. Oschkinat, Geometrical Representation of Coherence Transfer Selection by Pulsed Field Gradients in High-Resolution Nuclear Magnetic Resonance. J. Chem. Phys. 102 (1995), 3089-98.

14. J. R. Tolman and J. H. Prestegard, Homonuclear Correlation Experiments Using Pulsed-Field Gradients. Concepts Magn. Reson. 7 (1995), 247-62.

15. J.-M. Zhu and I. C. P. Smith, Selection of Coherence Transfer Pathways by Pulsed-Field Gradients in NMR Spectroscopy. Concepts Magn. Reson. 7 (1995), 281-91. 
Cambridge University Press

978-0-521-80696-1 - NMR Studies of Translational Motion

William S. Price

Frontmatter

More information

Preface

xiii

16. R. E. Hurd, Field Gradients \& Their Application. In Encyclopedia of Nuclear Magnetic Resonance, ed. D. M. Grant and R. K. Harris. vol. 3. (New York: Wiley, 1996), pp. 1990-2005.

17. T. Parella, High-Quality 1D Spectra by Implementing Pulsed-Field Gradients as the Coherence Pathway Selection Procedure. Magn. Reson. Chem. 34 (1996), 329-47.

18. W. S. Price, Gradient NMR. In Annual Reports on NMR Spectroscopy, ed. G. A. Webb. vol. 32. (London: Academic Press, 1996), pp. 51-142.

19. S. Berger, NMR Techniques Employing Selective Radiofrequency Pulses in Combination with Pulsed Field Gradients. Prog. NMR Spectrosc. 30 (1997), 137-56.

20. W. S. Price, Water Signal Suppression in NMR Spectroscopy. In Annual Reports on NMR Spectroscopy, ed. G. A. Webb. vol. 38. (London: Academic Press, 1999), pp. 289-354.

21. M. Weiger, T. Speck, and M. Fey, Gradient Shimming with Spectrum Optimisation. J. Magn. Reson. 182 (2006), 38-48.

22. W. S. Price, Pulsed Field Gradient NMR as a Tool for Studying Translational Diffusion, Part I. Basic Theory. Concepts Magn. Reson. 9 (1997), 299-336.

23. W. S. Price, Pulsed Field Gradient NMR as a Tool for Studying Translational Diffusion, Part II. Experimental Aspects. Concepts Magn. Reson. 10 (1998), 197-237. 


\section{Acknowledgements}

Many people have contributed to this book either directly or indirectly. I thank the authors whose work I have included. Particular thanks to my collaborators, colleagues and mentors over the years, including Dr Yuichi Aihara, Prof. Yoji Arata, Assoc. Prof. Janice Aldrich-Wright, Dr Alexander Barzykin, Prof. Paul Callaghan, Dr Reynaldo Castillo, Dr Bob Chapman, Dr Gary Dennis, Prof. István Furó, Prof. Kikuko Hayamizu, Prof. Lian-Pin Hwang, Prof. Masatsune Kainosho, Prof. Jorg Kärger, Prof. Philip Kuchel, Assoc. Prof. Andrew Shalliker, Prof. Olle Söderman, Dr Tim Stait-Gardner, Prof. Peter Stilbs, Dr Allan Torres and Prof. Sergey Traytak. The efforts of two of my PhD students, Mr Gang Zheng and Mr Nirbhay Yadav, for many hours spent proofreading are gratefully acknowledged. All errors that are contained in this book are, however, attributable only to me.

I also thank my editor, Dr Michelle Carey, who probably never thought that the book would ever be finished, and her editorial staff including Sarah Matthews.

Most of all I thank my wife, Hasna, and my two children, Edward and Roxanne, for their patience and support while I wrote this book. And to my late wonderful parents who always gave me every support and encouragement in life and also in pursuing an academic career.

The NSW State Government is acknowledged for financial support through a BioFirst Award. 


\section{Abbreviations and Symbols}

A

$a$

$\mathrm{ADC}$

$a_{M}$

$a_{\min }$

$b$

b

$\mathbf{B}_{0}$

$\mathbf{B}_{1}$

BPP

BSA

$C(Z, \Delta)$

CORE

COSY

CPMG

CTPG

\section{$D$}

$d$

$D_{i}$

$D_{i}^{0}$

$\langle D\rangle_{P}$

$\langle D\rangle_{W}$

$\langle D\rangle_{W}^{C}$ cross-sectional area of a sample tube

characteristic distance (e.g., radius of a pore or half the interplanar separation)

apparent diffusion coefficient (see also $D^{\text {app }}$ )

relaxation length

uncertainty in initial and final position during a gradient pulse

gradient or diffusion weighting factor

gradient or diffusion weighting vector

static magnetic field

radio frequency (rf) field

bipolar gradient pulses

bovine serum albumin

diffusion envelope

component resolved spectroscopy

correlation spectroscopy

Carr-Purcell-Meiboom-Gill sequence

constant time, pulse, and gradient and amplitude diffusion experiment

self-diffusion coefficient

embedding dimension

individual self-diffusion coefficient

self-diffusion coefficient of the $i^{\text {th }}$ oligomeric species at infinite dilution

population-weighted averaged diffusion coefficient

mass averaged diffusion coefficient

mass averaged diffusion coefficient including obstruction effects 
Cambridge University Press

978-0-521-80696-1 - NMR Studies of Translational Motion

William S. Price

Frontmatter

More information

xvi

D

D $^{\text {lab }}$

$\mathbf{D}_{\omega}$

$D_{\kappa}$

$D_{\omega}$

$D^{\infty}$

$D^{*}$

$D(t)$

$\mathbf{D}(t)$

$D^{0}$

$D_{\text {anion }}$

$D_{\text {cation }}$

$d_{\mathrm{f}}$

$d_{w}$

$D^{\text {app }}, D^{\text {app }}(t)$ or

$D^{\text {app }}(\Delta)$

$D^{\text {eff }}$

$D_{\text {eff }}^{*}$

$D_{\mathrm{b}}$

$D_{\mathrm{f}}$

$D_{\mathrm{i}}$

$D / D^{0}$

$\mathbf{D}_{\mathrm{M}}$

$D_{\mathrm{M}}$

DOSY

$D^{\text {pr }}$

DQ

$d_{\mathrm{s}}$

DCNMR

DDCOSY

DRCOSY

DDIF

DTI

DWI

$\Delta \bar{P}(\mathbf{R}, \Delta)_{\frac{1}{2}}$

E

$E, E(\mathbf{q}, \Delta)$
Abbreviations and Symbols

self-diffusion tensor

diffusion tensor in the laboratory axes frame $\left(x^{\prime}, y^{\prime}, z^{\prime}\right)$

diffusion coefficient spectrum or tensor

fractional diffusion coefficient

frequency-dependent diffusion coefficient

effective or long-time self-diffusion coefficient

asymptotic dispersion coefficient

(measuring time-dependent) diffusion coefficient obtained in the SGP limit

time-dependent diffusion tensor

infinite dilution (or short time) self-diffusion coefficient

anion diffusion coefficient

cation diffusion coefficient

dimension of the fractal space

random walk dimension

'apparent diffusion coefficient' obtained by simplistically

applying the free diffusion solution to the analysis of a more

complicated system

synonymous with $D^{\infty}$

effective time-dependent dispersion coefficient

bound ligand diffusion coefficient

free ligand diffusion coefficient

'distinct' diffusion coefficient

relative diffusion coefficient

mutual diffusion tensor

mutual diffusion coefficient

diffusion ordered spectroscopy

self-diffusion tensor in the principal axes frame $(x, y, z)$

double quantum

spectral dimension

see electrophoretic NMR

diffusion-diffusion correlation spectroscopy

diffusion-relaxation correlation spectroscopy

diffusion decay in the internal field

diffusion tensor imaging

diffusion-weighted imaging

average propagator width at half-height

elliptic integral of the second kind

spin-echo attenuation (normally synonymous with $E_{\text {Diff) }}$ 
Cambridge University Press

978-0-521-80696-1 - NMR Studies of Translational Motion

William S. Price

Frontmatter

$\underline{\text { More information }}$

$E_{\mathrm{dc}}$

$E_{\text {Diff }}$

$E\left(\mathrm{~g}_{1}\right)$

$E(\mathbf{q}, \infty)$

$E_{\text {phase }}$

$E_{\text {poly }}$

$E_{\text {Relax }}$

ENMR

F

$\operatorname{FT}\{L(Z)\}$

$f$

$\mathrm{F}_{1}$

$\mathrm{F}_{2}$

$f_{\mathrm{s}}$

FID

FT

g

$\mathbf{g}_{0}$

$g^{\text {app }}$

$g_{\mathrm{e}}$

$g_{\text {eff }}$

$\mathbf{g}_{\text {int }}$

$g_{\mathrm{r}}$

$\mathrm{G}_{x, y, z}$

GPD

H

$H$

$h$

HMQC

HRMAS

HSA

$\mathfrak{I}$

$\mathbf{i}, \mathbf{j}, \mathbf{k}$

IMFG

$\mathbf{J}_{A}(t)$ pulsed electric field

spin-echo attenuation due to diffusion

spin-echo attenuation due to $B_{1}$ gradients (normally synonymous with $\left.E_{\text {Diff }}\right)$

long-time diffusive attenuation

signal attenuation due to the phase-twist or residual phase-twist spin-echo attenuation in a polydisperse system

spin-echo attenuation due to relaxation

electrophoretic NMR

Perrin factor

reciprocal lattice

friction coefficient

indirectly detected dimension in a 2D NMR experiment

directly detected dimension in a 2D NMR experiment

free volume contributed by the solvent

free induction decay

Fourier transform

magnetic field gradient (applied) - normally spatially homogeneous (i.e., constant)

background or constant magnetic gradient

initial guess of gradient strength

encoding gradient

effective gradient

magnetic gradient arising from internal susceptibility differences (see background (magnetic field) gradients)

read gradient

gradient used for spatial localisation in MRI

Gaussian phase distribution

mean square displacement tensor

Hurst exponent

reduced permeability $(=M a / D)$

heteronuclear multiple-quantum coherence

high-resolution magic angle spinning

human serum albumin

tortuosity

unit coordinate vectors

internal magnetic field gradient (see background (magnetic field) gradients)

flux associated with species $A$ at time $t$ 
Cambridge University Press

978-0-521-80696-1 - NMR Studies of Translational Motion

William S. Price

Frontmatter

More information

xviii

Abbreviations and Symbols

$J_{n}(x)$

$j_{n}(x)$

K

K

K

$\mathbf{k}($ or $k)$

$k$

$k_{1}$

$k_{+1}$

$k_{-1}$

$K_{\mathrm{d}}$

$K_{\text {e }}$

$l$

$L(Z)$

$\mathcal{L}$

LED

M

$M_{0}$

MAGROFI

MGSE

MAS

MMME

MOSY

MRI

MSD, $\left\langle R^{2}\right\rangle$

MW

$M_{\mathrm{n}}$

$M_{\mathrm{w}}$

LED

n

$n$

$n_{\text {avg }}$

$n\left(R_{\text {cell }}\right)$

$\mathrm{NOE}$

NOESY

$O_{D}$
Bessel function of the first kind of order $n$

spherical Bessel function of order $n$

modified Bessel function of the third kind

elliptic integral of the first kind

mobility factor

dephasing strength of gradients when used in imaging (see $\mathbf{k}$, $k$-space)

gradient or diffusion weighting factor, more commonly written as $b$

first-order rate constant

(forward) first-order rate constant

(reverse) first-order rate constant

dissociation constant

equilibrium constant

step length or size, length of sample tube, length of receiver coil

lattice correlation function

Laplace transform

longitudinal eddy current delay

relaxivity (or permeability)

thermal equilibrium magnetisation

magnetisation rotating frame imaging

Modulated Gradient Spectroscopy see OGSE

magic angle spinning

multiple modulation multiple-echo

mobility ordered spectroscopy

(nuclear) magnetic resonance imaging

mean square displacement

molecular weight

number-average molecular weight

weight-average molecular weight

longitudinal eddy current delay

outward surface normal

number of spatial dimensions

average number concentration of counterions in the spherical shell

number concentration of counterions at the outer shell boundary nuclear Overhauser effect

nuclear Overhauser enhancement spectroscopy

obstruction factor 
OGSE

$p$

$P_{\mathrm{b}}$

$P_{\mathrm{e}}$

$P_{\mathrm{f}}$

$P_{n}(x)$

$\bar{P}(\mathbf{R}, t)$

$P(a)$

$P(x, y)$

$P(v)$

$P\left(\mathbf{r}_{0}, \mathbf{r}_{1}, t\right), P$

$P_{\phi}, P_{\phi}(\phi, t)$

$[\mathrm{P}]_{\text {Total }}$

PFG

PGSE

PGSTE

q

$\Delta \mathbf{q}$

$q_{\max }$

$\mathbf{r}$

$\Delta \mathbf{r}$

$\mathbf{r}_{0}$

$\mathbf{r}_{1}$

$r_{\mathrm{S}}$

$\mathbf{R}$

$R_{\mathrm{a}}$

$R_{\text {cell }}$

$R_{\mathrm{S}}$

RMS

RMSD

RTOP

RTOP $^{0}$

RTOP $^{\mathrm{e}}$

rf

$S(\mathbf{q})$ oscillating gradient spin-echo (sequence)

coherence order (see also multiple quantum coherences)

bound (fractional) population (of a ligand)

Péclet number

free (fractional) population (of a ligand)

Legendre polynomial of order $n$

average or mean propagator, the probability that a particle will move a distance $\mathbf{R}$ in time $t$

distribution sphere radii

joint probability (e.g., $\left.P\left(D_{i i}, D_{j j}\right), P(\omega, D)\right)$

distribution of velocities

diffusion propagator, the probability of moving from $\mathbf{r}_{0}$ to $\mathbf{r}_{1}$ in time $t$

probability of phase distribution $=$ phase distribution

function

total macromolecule concentration

pulsed field gradient

pulsed gradient spin-echo

pulsed gradient stimulated (spin) echo

dephasing strength of gradients when used for measuring dis-

placement (see q, $q$-space)

gradient mismatch

maximum value of $q$ used in an experiment

position of a particle (or voxel)

sample movement

initial position

finishing position

Stokes radius (effective hydrodynamic radius)

dynamic displacement $\left(=\mathbf{r}_{1}-\mathbf{r}_{0}\right)$

Rayleigh number

radius of an electroneutral spherical shell

radius of a charged hard sphere

root mean square

root mean square displacement

return to origin probability

RTOP in free isotropic solution

dimensionless RTOP enhancement

radio frequency

Fourier transform of $\rho\left(\mathbf{r}_{\mathbf{1}}\right)$, signal in MRI 
Cambridge University Press

978-0-521-80696-1 - NMR Studies of Translational Motion

William S. Price

Frontmatter

More information

$\mathrm{xX}$

$\left|S(\mathbf{q})^{2}\right|$

$\left|S_{0}(\mathbf{q})^{2}\right|$

SE

SGP

SGSE

$S_{\mathrm{P}}$

$S_{\mathrm{P}} / V_{\mathrm{P}}$

STE

STRAFI

$t_{\mathrm{e}}$

$t_{\text {echo }}$

$T$

$T$

$T_{1}$

$T_{2}$

$T_{2}^{Z Q C}$

$t_{\mathrm{r}}$

$T_{\mathrm{RD}}$

$T \mathrm{~s}$

TOCSY

\section{u}

$v$

$v$

$v^{+}$

$v^{-}$

$v_{\max }$

$v_{0}$

V

$V_{\mathrm{h}}$

$V_{\mathrm{P}}$

$V_{\mathrm{s}}$

V

$\mathbf{v}_{i}(t)$

$\overline{\mathbf{V}}$

$\langle v\rangle$

$\left\langle v^{2}\right\rangle$

$\langle v(0) v(t)\rangle$
Abbreviations and Symbols

power spectrum of $\rho\left(\mathbf{r}_{\mathbf{0}}\right)$, elastic incoherent structure factor or form factor

average pore structure factor

spin-echo (sequence) (or Hahn echo (sequence))

short gradient pulse approximation

steady gradient spin-echo

surface area of a pore

surface (area)-to-volume ratio of a pore

stimulated echo (sequence)

stray-field imaging

delay for eddy current dissipation

time at which echo formation occurs

temperature

total time for image acquisition

spin-lattice relaxation time

spin-spin relaxation time

transverse relaxation time of a zero-quantum coherence

reptation time

radiation damping time constant

singlet relaxation time

total correlation spectroscopy

fluctuating part of velocity

velocity

kinematic viscosity

velocity of cationic species

velocity of anionic species

maximum measurable velocity (see NMR Imaging - max measurable flow rate)

constant velocity

total volume (of a system)

partial specific volume of a solvent

volume of a pore

partial specific volume of a solute

(local) spin velocity (see also Lagrangian velocity field)

velocity of a particle in the barycentric reference frame

average velocity

average velocity

mean square velocity

velocity auto-correlation function 
Cambridge University Press

978-0-521-80696-1 - NMR Studies of Translational Motion

William S. Price

Frontmatter

More information

WATERGATE

$x, y, z$

$(r, \theta, \phi)$

$z_{0}$

$z_{1}$

$Z$

$\langle Z\rangle$

$\left\langle Z_{u}^{2}\right\rangle$

$Z_{\mathrm{u}}(t)$

$\left\langle Z^{n}(\Delta)\right\rangle$ a gradient-based water suppression technique

Cartesian coordinates (primes can be used to denote the laboratory frame, if required)

spherical polar coordinates

initial position for a one-dimensional motion

finishing position for a one-dimensional motion

one dimensional dynamic displacement $\left(=z_{1}-z_{0}\right)$

average displacement

positional variance

displacement fluctuation

moments of the average propagator $(\bar{P}(Z, \Delta))$

\section{Greek}

$\alpha$

$\alpha$

$\Gamma(\Delta)$

$\gamma$

$\gamma$

$\gamma_{\text {eff }}$

$\delta$

$\delta_{\mathrm{h}}$

$\delta_{\mathrm{nm}}$

$\Delta$

$\Delta$

$\Delta_{\mathrm{CPMG}}$

$\Delta^{\text {eff }}$

$\eta$

$\eta$

$\eta_{\mathrm{F}}$

$\Theta$

$\theta$

$\theta_{\mathrm{a}}$

$\kappa$

$\kappa$

$\kappa$

$\Lambda$

$\Lambda_{q}$ thermal expansion

time-independent scaling constant

time-dependent function characteristic of a geometry

(chemical) activity coefficient

gyromagnetic ratio

effective gyromagnetic ratio

duration of the gradient pulse

hydration/solvation (grams of solvent/grams of solute)

Kronecker delta

difference

timescale of the diffusion measurement

effective diffusion time (i.e., effective $\Delta$ ) in a CPMG measurement effective diffusion time (i.e., effective $\Delta$ ) in an OGSE experiment magic (gradient) ratio

viscosity

filling factor

Spectral function

occupation probability

exponent of anomalous diffusion

$=2 / d_{w}$

thermal diffusivity

exponent characterising the time dependence of the mean square

displacement

conductivity

pitch of a magnetisation helix due to a pulse of area ' $2 \pi q$ ' 
Cambridge University Press

978-0-521-80696-1 - NMR Studies of Translational Motion

William S. Price

Frontmatter

More information

xxii

Abbreviations and Symbols

$\begin{array}{ll}\rho\left(\mathbf{r}_{0}\right), \rho\left(\mathbf{r}_{0}, 0\right) & \text { equilibrium spin density } \\ \lambda & \text { separation between adjacent sites } \\ \mu & \text { drift velocity } \\ \mu_{0} & \text { permittivity constant (magnetic permeability) } \\ \sigma & \text { length of oscillating gradient pulse } \\ \sigma & \text { standard error } \\ \tau & \text { a delay in a pulse sequence or discretisation time } \\ \tau_{\mathrm{b}} & \text { lifetime of a ligand in the bound state } \\ \tau_{\mathrm{c}} & \text { reorientational correlation time } \\ \tau_{\mathrm{e}} & \text { extracellular (external to the pore) lifetime } \\ \tau_{\mathrm{f}} & \text { lifetime of a ligand in the free state } \\ \tau_{\mathrm{i}} & \text { intracellular (internal to the pore) lifetime } \\ \tau_{\mathrm{J}} & \text { time between jumps } \\ \tau_{\mathrm{m}} & \text { mixing time } \\ \tau_{v} & \text { velocity correlation time } \\ \nu & \text { frequency (with respect to spectrometer reference frequency) } \\ v_{l} & \text { step rate } \\ \Delta v & \text { linewidth } \\ \Delta v_{1 / 2} & \text { linewidth at half-height } \\ \xi & \text { dimensionless variable (= } D \Delta / a^{2} \text { ) for characterising restricted } \\ \Phi_{\text {Flow }} & \text { diffusion } \\ \phi & \text { change in phase of a spin-echo signal due to flow } \\ \phi & \text { phase angle (phase of spins) } \\ \phi & \text { porosity } \\ \left\langle\phi^{2}\right\rangle & \text { volume fraction } \\ \omega & \text { mean square phase distribution } \\ & \text { frequency (rad s }{ }^{-1} \text { ) }\end{array}$

